\title{
Calibration of the two microphone transfer function method with hard wall impedance measurements at different reference sections
}

\author{
René Boonen ${ }^{\mathrm{a}, *}$, Paul Sas ${ }^{\mathrm{a}}$, Wim Desmet ${ }^{\mathrm{a}}$, Walter Lauriks ${ }^{\mathrm{b}}$, Gerrit Vermeir ${ }^{\mathrm{b}}$ \\ ${ }^{a}$ K.U.Leuven, Department of Mechanical Engineering, P.M.A., Celestijnenlaan 300B, B-3001 Leuven, Belgium \\ ${ }^{\mathrm{b}}$ K.U.Leuven, Laboratory for Acoustics and Thermal Physics, Celestijnenlaan 200D, B-3001 Leuven, Belgium
}

\section{A R T I C L E I N F O}

\section{Article history:}

Received 6 August 2008

Received in revised form

27 November 2008

Accepted 1 December 2008

Available online 10 December 2008

PACS:

43.58.Bh

43.58.Vb

43.64.Yp

Keywords:

Acoustic impedance

Two-microphone-method

Calibration

Hard wall impedance

\begin{abstract}
A B S T R A C T
In many acoustic simulations, particularly when using lumped parameter models or electrical analog circuits, the acoustic impedance of a component needs to be determined accurately. A widely used acoustic impedance measurement method is the "two microphone transfer function method", which is standardized in ISO-10534-2. When the acoustic impedance is needed over a wide frequency band with a high impedance magnitude range, this method faces some limitations. In this paper, a calibration method is proposed which uses hard wall impedance measurements at different positions of the reference section. The measured hard wall impedance is used to calibrate the microphone positions, to compensate the microphone mismatch and to estimate the wave guide damping. Also, the measured hard wall impedance can be used as performance criterion. It can be used to select frequency bands from different load impedance measurements where the accuracy is maximum and to assemble them in a load impedance measurement. As a result, impedances with a high ratio with respect to the characteristic duct impedance can be accurately measured. The capability of the presented calibration method is illustrated by the impedance measurement of an open duct end and a closed tube.
\end{abstract}

(c) 2008 Elsevier Ltd. All rights reserved.

\section{Introduction}

A proper design of acoustic systems that involve components such as silencers, resonators, absorbing materials, horns, etc., requires an accurate acoustic characterization of the various components. Such systems can be simulated using lumped element models or electrical analog circuits. The accuracy of the simulation results depends on the accuracy of the acoustic impedance of each component. Therefore, accurate acoustic impedance measurement methods are mandatory.

In general, three approaches for acoustic impedance measurement are available. These are: direct methods using the pressure particle velocity ratio, indirect methods by comparing the target impedance with calibration impedances and the wave reflection methods.

The direct method uses a pressure and a particle or volume velocity sensor [1-3], whereby the impedance is calculated directly from the ratio between both measured quantities. The volume velocity sensor can be eliminated in some situations by applying a calibrated volume velocity source. This approach works well at low frequencies, until the relative distances between the sensors reach the order of magnitude of a half wave length. The measured impedance is composed by the

\footnotetext{
* Corresponding author. Tel.: +32 16322536; fax: +32 16322987

E-mail address: rene.boonen@mech.kuleuven.be (R. Boonen).
} 
impedances of the objects present in the environment of the sensor. The impedance of the object under investigation has to be separated from the measured impedance.

The second approach is based on connecting known impedances to the impedance to be measured. These methods are commonly used to determine the internal acoustic impedance of a source [4-6]. To the impedance to be measured, known acoustic loads are connected and the response to the source is measured. A set of equations results, from which the unknown impedance can be determined.

The third approach is to determine the impedance from wave reflection. In the wave reflection technique, waves are radiated towards the sample, whereupon the waves are reflected. From the measurement of the incident and reflected waves, the reflection coefficient and the corresponding acoustic impedance are determined. The target impedance can be measured at a position different from the sensor positions. The method is suitable for the high frequency range, where wave behaviour is fully developed.

The two standardized wave reflection methods are the standing wave ratio (SWR) method and the two microphone transfer function method.

This SWR (the classical Kundt duct) method determines the acoustic impedance from pressure measurements of the standing wave pattern in a duct. The method is described in the ISO-10534-1 standard [7]. The ends of the Kundt duct are closed by an excitation source at one side and the unknown impedance at the other side. The source generates a sinusoidal signal which results in a standing wave pattern in the duct. A microphone is moved along the axis of the duct. The minimum and maximum pressure amplitude of the standing wave and the location where the minimum and maximum amplitude occur are determined. From these data, the reflection coefficient and the acoustic impedance are calculated.

The "two microphone transfer function method", which is described in ISO-10534-2 [7], has outcasted the SWRmethod. The two microphone transfer function method has several advantages compared to the SWR-method. The mechanical construction of the measurement setup is simpler. The frequency band is broadened towards low frequencies. The measurements can be carried out below the first wave guide resonance up to until cross-section resonance occurs.

This method uses the transfer functions measured between two pressure sensors at two distinct positions in the measurement wave guide to determine the acoustic impedance attached at one side of the wave guide. The method is discussed in more detail in the next section.

When the acoustic impedance is needed over a wide magnitude range over a wide frequency band, the standardized impedance measurement methods face limitations. Several error mechanisms has been investigated [3,8,9]. Bodén and Åbom [8] treat the bias and random errors which occur when the transfer function between the microphones has been estimated. The conclusion was that the duct length should be kept small, the source end should be non-reflective and the first microphone should be as close as possible to the sample. The effect of the spacing between the microphones was also investigated. The maximum spacing determines the frequencies where singularities occur, i.e. where a half wave length stands between the microphone positions. Also, large errors occur when pressure nodes are present at the microphone locations. The minimum spacing is determined by the phase error sensitivity which occur when the wave-numbermicrophone-distance-product tends to zero.

Katz [9] presents a method to find the microphone positions with improved accuracy. The wave guide will be closed by a rigid steel plate wherein a supplementary microphone is positioned. From the transfer function between a measurement microphone and the closed end microphone, its distance to the closed end can be estimated with higher precision than the standard ISO-method using the ruler. This action reduces the phase error sensitivity of the measurement setup.

Dickens et al. [3] present a calibration method for a three microphone setup to measure the acoustical impedance of musical instruments such as flutes. He uses three non-reflecting calibration loads and spectral shaping to keep the acoustic power constant at the reference section in the frequency band of interest which situates between $150 \mathrm{~Hz}$ and $4 \mathrm{kHz}$. No assumption was made about the microphone characteristics or the exact geometry of the impedance head. For a two microphone setup, he estimates that the calibration method using tree non-reflective loads has good performance in a frequency range of 2-3 octaves, depending on the required precision.

Gibiat and Laloë [10] present a TMTC (two-microphone-three-calibration) method also for the measurement of the acoustical impedance of musical instruments. The experimental setup consists of a measurement head made of brass with $15 \mathrm{~mm}$ bore diameter and $7 \mathrm{~mm}$ wall thickness. To calibrate the setup, three devices with known impedance are subsequently connected. The first one is a hard wall closure at the reference section, the other two are two cavities. At each calibration impedance, the microphone transfer function will be measured. The impedance to be measured will then be expressed in terms of the three calibration impedances and the four measured transfer functions.

There lacks a performance criterion for a calibration method and measurement setup, on which the expected performance can be checked when an arbitrary unknown load impedance is connected to the measurement setup. As the performance of the impedance measurement varies widely in terms of frequency, such a criterion can be used to select the frequency bands from different measurements where they have maximum accuracy. In this way, the frequency band and the impedance magnitude range can be extended.

In this paper, a calibration method is proposed which is based on hard wall impedance measurements at different positions of the reference section. The reference section is shifted by connecting closed duct ends with the same crosssection as the measurement duct to the measurement duct end. As a result, the hard wall is displaced by a distance represented by the length of the duct ends. The distances between the microphones and the respective reference sections will be expressed as wave travelling times. As a result, the speed of sound is eliminated from the calibration procedure. 
The wave travelling time from each microphone position to the respective hard wall terminations will be determined by measuring the frequency at which a quarter wave length stands between the respective microphone positions and the reference section. In this way, manufacturing errors in the length of the calibration duct ends and the deviation of the position of the acoustic centre of the microphones are eliminated. The measured transfer functions between the microphones of the calibration duct ends are used to eliminate the sensor mismatch and to estimate the measurement wave guide damping. Then, the transfer function between the microphones is measured with the unknown impedance at the duct end. The unknown impedance will be determined in terms of the calibration transfer functions, the corresponding wave travelling times and the wave guide damping.

The magnitude of the measured hard wall impedance is used as a performance criterion of the setup and the calibration. A low value will indicate that the reability of the measured unknown impedance will be low. The hard wall impedance can also be used as a selection criterion between measurements at different reference sections. In this way, frequency bands wherein pressure nodes occur at the microphone position, which cause a drop in the impedance magnitude, will be replaced by parts of other measurements with correct impedance data. The outcome is an impedance measurement method which is capable to cover two decades in frequency $(10 \mathrm{~Hz}-1 \mathrm{kHz})$ with a high measurement range $(50-100 \times$ the characteristic wave guide impedance) in magnitude.

\section{The two microphone transfer function method}

This section discusses the principle of the two microphone transfer function method including wave guide damping. Fig. 1 presents the setup for acoustic impedance measurement. The setup consists of a straight duct which is the measurement acoustic wave guide. At the left end, an excitation source, such as a loudspeaker, is connected. At the right end, the impedance to be measured is connected. This impedance includes everything present at the right side of the reference section. Two microphones at two distinct positions $x_{1}$ and $x_{2}$ measure the sound pressure inside the duct. From the transfer function between the two microphones, the reflection coefficient and consequently, the unknown connected impedance will be determined.

Wave attenuation is caused by three different mechanisms. These are wall friction, heat exchange and internal gas viscosity [11]. The wave guide dimensions determine the dominant attenuation mechanism. In the frequency domain, the wave guide damping can be approximated by introducing a constant loss factor $\xi$ in the compressibility $\kappa$ of the medium [12]:

$$
\kappa \approx \kappa_{0}(1+\mathrm{j} 2 \xi)
$$

wherein $\mathrm{j}=\sqrt{-1}$ and $\kappa_{0}$ is the lossless compressibility of the medium. This type of loss factor can be taken as frequency independent in the measured frequency domain. This damping mechanism provides a good fit with the measured transfer functions in a wide frequency range, as will be demonstrated in Fig. 7. The effect of the loss factor $\xi$ on the characteristic impedance $Z_{0}$ of the wave guide and the propagation constant $\gamma$ is

$$
Z_{0} \approx \sqrt{\frac{\rho_{0} \kappa_{0}}{S^{2}}}(1+\mathrm{j} \xi) \text { and } \gamma \approx \omega \sqrt{\frac{\rho_{0}}{\kappa_{0}}}(1-\mathrm{j} \xi)
$$

wherein $\omega$ is the angle frequency, $\rho_{0}$ is the density of the medium and $S$ is the cross-section of the wave guide.

The wave pattern in the wave guide is governed by the one-dimensional Helmholtz wave equation, which describes the pressure distribution along the wave guide. At each position $x$, the pressure in terms of the propagation constant $\gamma$ in the wave guide equals [13]

$$
p(x, \gamma)=\phi_{g} \frac{Z_{0} Z_{g}}{Z_{0}+Z_{g}} \frac{\mathrm{e}^{-\mathrm{j} \gamma l}}{1-\Gamma_{l} \Gamma_{g} \mathrm{e}^{-\mathrm{j} 2 \gamma l}}\left(\mathrm{e}^{\mathrm{j} \gamma x}+\Gamma_{l} \mathrm{e}^{-\mathrm{j} \gamma x}\right)
$$

wherein $\phi_{g}$ is the source volume velocity, $Z_{g}$ the source internal impedance, $l$ the distance between the exciting sound source and the reference section, $\Gamma_{l}$ and $\Gamma_{g}$ the reflection coefficients at the load side and source side, respectively.

The source volume velocity $\phi_{g}$ splits over the source internal impedance $Z_{g}$ and the wave guide characteristic impedance $Z_{0}$. The volume velocity flowing through the wave guide reflects alternately at the load impedance $Z_{l}$ with a

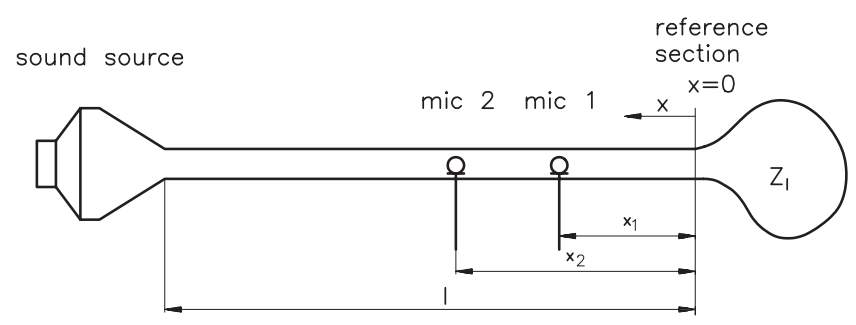

Fig. 1. Wave guide with an unknown acoustic impedance $Z_{l}$. 
reflection coefficient $\Gamma_{l}$ and at the source impedance $Z_{g}$ with a reflection coefficient $\Gamma_{g}$. As $\Gamma_{l}<1$ and $\Gamma_{g}<1$, the sum of the series of reflections is finite and equals the factor $\mathrm{e}^{-\mathrm{j} \gamma l} /\left(1-\Gamma_{l} \Gamma_{\mathrm{g}} \mathrm{e}^{-\mathrm{j} 2 \gamma l}\right)$. The factor $\left(\mathrm{e}^{\mathrm{j} \gamma x}+\Gamma_{l} \mathrm{e}^{-\mathrm{j} \gamma x}\right)$ describes the pressure distribution in the duct in terms of the position $x$.

To measure the load impedance using the two microphone method, the transfer function $T_{12}$ between the pressures at two distinct positions $x_{1}$ and $x_{2}$ is taken:

$$
T_{12}=\frac{p\left(x_{1}, \gamma\right)}{p\left(x_{2}, \gamma\right)}=\frac{\mathrm{e}^{\mathrm{j} \gamma x_{1}}+\Gamma_{l} \mathrm{e}^{-\mathrm{j} \gamma x_{1}}}{\mathrm{e}^{\mathrm{j} \gamma x_{2}}+\Gamma_{l} \mathrm{e}^{-\mathrm{j} \gamma x_{2}}}
$$

Notice that the source reflection coefficient drops out, the reflection coefficient at the load is the single unknown. Consequently, the choice of the source type is free. The load reflection coefficient $\Gamma_{l}$ will then be isolated from Eq. (4) and the load impedance $Z_{l}$ results from

$$
Z_{l}=Z_{0} \frac{1+\Gamma_{l}}{1-\Gamma_{l}}=\mathrm{j} Z_{0} \frac{\sin \gamma x_{1}-T_{12} \sin \gamma x_{2}}{\cos \gamma x_{1}-T_{12} \cos \gamma x_{2}}
$$

\section{Improved calibration method}

Ideally, the impedance of a hard wall at the reference section is infinite. In the laboratory, the hard wall impedance will always be finite due to the measurement imperfections. The magnitude of the measured hard wall impedance can be used as a quality criterion for the setup and the calibration procedure. The design of the setup and the calibration procedure should result in a closed end impedance as high as possible. After calibration, the unknown impedance can be connected to the reference section. The range in which the unknown impedance can be measured depends on the magnitude of the measured hard wall impedance.

The laboratory setup, used to investigate the calibration method, is presented in Fig. 2. It consists of a thick-walled steel duct with $40 \mathrm{~mm}$ internal diameter and $1.5 \mathrm{~m}$ length. At the back end situates a $60 \mathrm{~W}$ horn driver. Two PCB-106B pressure sensors are positioned at a distance $x_{1}=0.3 \mathrm{~m}$ and $x_{2}=0.47 \mathrm{~m}$ from the reference section. An HP-3562 signal analyser excites the horn driver with a stepped sine signal and measures the transfer function between the two sensors, from which the acoustic impedance connected to the reference section will be determined.

To calibrate the setup, the transfer function will be measured with the wave guide closed at the reference section. Thereafter, the reference section will be shifted to another position by interconnecting a short wave guide. A new transfer function will be measured. This procedure can be repeated several times with different lengths of interconnected wave guides. From the obtained transfer functions, the wave guide will be calibrated. This is carried out by determining the hard wall impedance of the closed wave guide. Theoretically, the reflection coefficient $\Gamma=1$. As observed from expression (5), the hard wall impedance is very sensitive to errors, resulting in low magnitude of the hard wall impedance. The calibration has to minimize the errors. Consequently, it results in the maximum hard wall impedance magnitude. At least two transfer functions are necessary to calibrate the setup.

In this investigation. three transfer functions are used. The first one, $T_{12}$, is measured with the reference section at $x_{1}=0.3 \mathrm{~m}$ and $x_{2}=0.47 \mathrm{~m}$, as presented in Fig. 3. The second one, $T_{34}$, is measured with the reference section shifted $47 \mathrm{~mm}$, so $x_{3}=0.347 \mathrm{~m}$ and $x_{4}=0.517 \mathrm{~m}$, as presented in Fig. 4 . The third one, $T_{56}$, is measured with the reference section shifted $302 \mathrm{~mm}$, so $x_{5}=0.602 \mathrm{~m}$ and $x_{6}=0.779 \mathrm{~m}$.

The following steps in the calibration procedure will be performed.

- The distances between the microphones and the reference section are expressed in terms of wave travelling times. In this way, the speed of sound is eliminated from the calibration process.

- The sensor mismatch will be eliminated.

- The wave guide damping will be estimated.

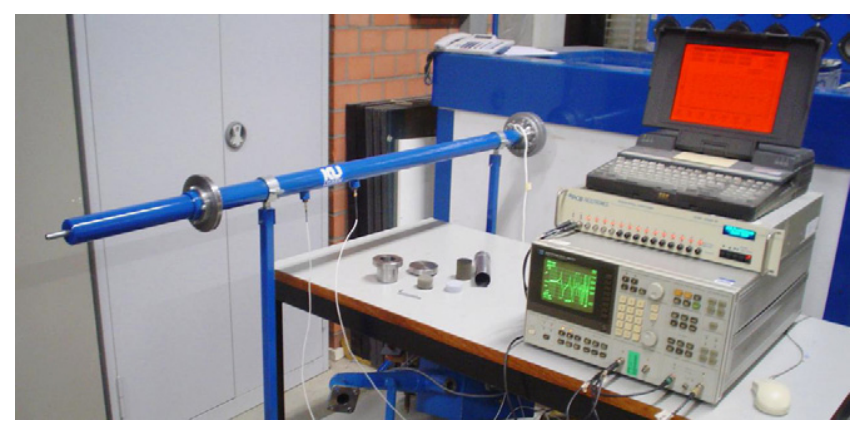

Fig. 2. Laboratory setup for acoustic impedance measurement. 


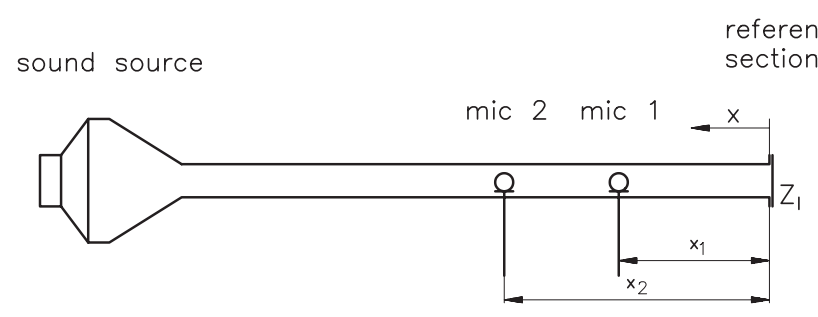

Fig. 3. Calibration setup with duct end closed at the reference section.

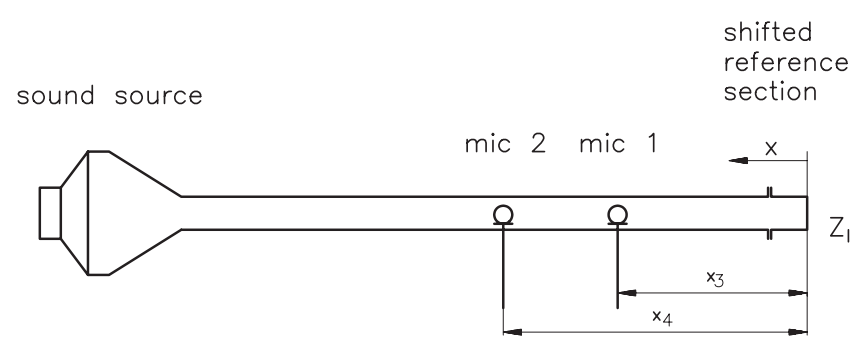

Fig. 4. Calibration setup with duct end closed at the shifted reference section.

- The singularities occurring in the frequency band of interest can be removed using additional transfer functions at other reference sections.

\subsection{Elimination of the speed of sound}

The aim of the elimination of the speed of sound from the calibration procedure is to avoid the measurement of the distance between the acoustic centres of the microphones, the ambient pressure and the temperature. In this way, deviations in these measured values are eliminated from the calibration procedure.

The distances $x_{1}$ and $x_{2}$ in expression (5) can be substituted by the travelling times $t_{1}$ and $t_{2}$ needed for the wave to travel from these positions to their reference sections, respectively. The relation between the travelling times and their respective distances is

$$
t_{1}=\frac{x_{1}}{v_{\mathrm{ph}}} \text { and } t_{2}=\frac{x_{2}}{v_{\mathrm{ph}}}
$$

wherein $v_{\mathrm{ph}}=\omega / \operatorname{Re}(\gamma)$ is the phase velocity of the sound. The resulting transfer function $T_{12}$ between the two microphone voltages in terms of travelling times will be

$$
T_{12}=\frac{\delta\left(Z_{c} \cos \beta t_{1}+\mathrm{j} Z_{0} \sin \beta t_{1}\right)}{\left(Z_{c} \cos \beta t_{2}+\mathrm{j} Z_{0} \sin \beta t_{2}\right)}
$$

wherein $Z_{c}$ is the measured impedance of the closed wave guide and $\beta=\omega(1-\mathrm{j} \xi)$. The microphone mismatch $\delta$ results from the conversion of the measured pressure to microphone voltage.

The travelling times $t_{1}$ and $t_{2}$ are estimated from the measured transfer function $T_{12}$, as presented in Fig. 5. They correspond to the first pole for the most far microphone and the first zero for the nearest microphone position. The pole and the zero correspond to the first node of the pressure distribution of the standing wave appearing at the positions $x_{1}$ and $x_{2}$, respectively. The travelling times $t_{1}$ and $t_{2}$ equal

$$
t_{1}=\frac{1}{4 f_{1}} \quad \text { and } \quad t_{2}=\frac{1}{4 f_{2}}
$$

in which the frequencies $f_{1}$ and $f_{2}$ are associated to the frequencies determined by the quarter wave length between the reference section and the positions $x_{1}$ and $x_{2}$, respectively.

This procedure is applied to all the measured transfer functions. For the transfer function $T_{34}$, where $x_{3}$ takes the place of $x_{1}$ and $x_{4}$ the place of $x_{2}$, the travelling times will be

$$
t_{3}=\frac{1}{4 f_{3}} \quad \text { and } \quad t_{4}=\frac{1}{4 f_{4}}
$$

wherein the frequencies $f_{3}$ and $f_{4}$ correspond to the quarter wave lengths between the positions $x_{3}$ and $x_{4}$ and the reference section, respectively. 


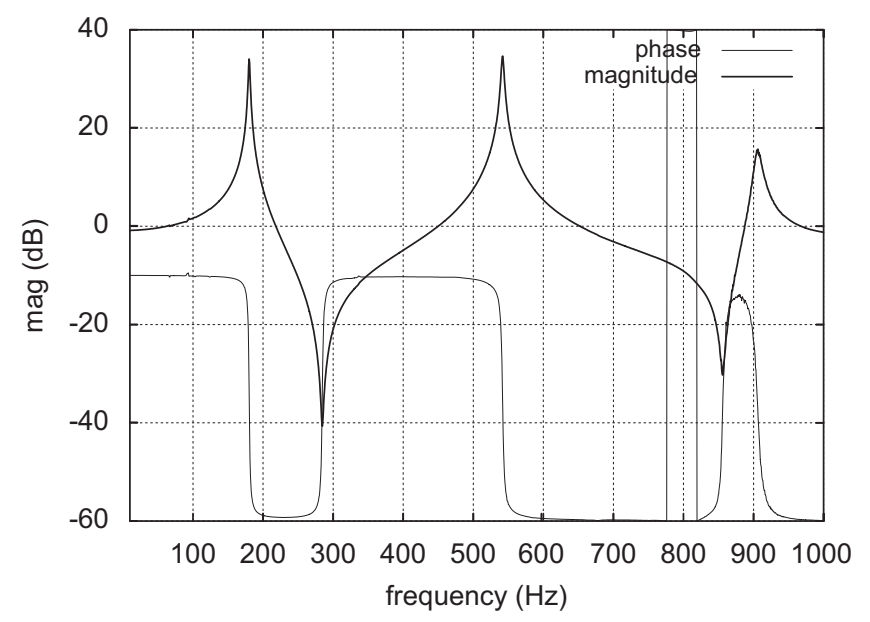

Fig. 5. Measured transfer function $T_{12}$ between the sensors located at $x_{1}=0.3 \mathrm{~m}$ and $x_{2}=0.47 \mathrm{~m}$ with the duct closed at the reference section.

Similar for the transfer function $T_{56}$, where $x_{5}$ takes the place of $x_{1}$ and $x_{6}$ the place of $x_{2}$, the travelling times will be

$$
t_{5}=\frac{1}{4 f_{5}} \quad \text { and } \quad t_{6}=\frac{1}{4 f_{6}}
$$

wherein the frequencies $f_{5}$ and $f_{6}$ correspond to the quarter wave lengths between the positions $x_{5}$ and $x_{6}$ and the reference section, respectively.

\subsection{Elimination of the microphone mismatch}

The electrical transfer functions between pressure and voltage of the microphones have to be eliminated. By considering the ratio $T_{c 1}$ between the two measured transfer functions $T_{12}$ and $T_{34}$, the sensor mismatch $\delta$ vanishes from numerator and denominator:

$$
\begin{aligned}
& T_{c 1}=\frac{T_{12}}{T_{34}}=\frac{\delta\left(Z_{c 1} \cos \beta t_{1}+\mathrm{j} Z_{0} \sin \beta t_{1}\right)}{\left(Z_{c 1} \cos \beta t_{2}+\mathrm{j} Z_{0} \sin \beta t_{2}\right)} \cdot \frac{\left(Z_{c 1} \cos \beta t_{4}+\mathrm{j} Z_{0} \sin \beta t_{4}\right)}{\delta\left(Z_{c 1} \cos \beta t_{3}+\mathrm{j} Z_{0} \sin \beta t_{3}\right)} \\
& T_{c 1}=\frac{z_{c 1}^{2} \cos \beta t_{1} \cos \beta t_{4}-\sin \beta t_{1} \sin \beta t_{4}+\mathrm{j} z_{c 1} \sin \beta t_{1} \cos \beta t_{4}+\mathrm{j} z_{c 1} \cos \beta t_{1} \sin \beta t_{4}}{z_{c 1}^{2} \cos \beta t_{2} \cos \beta t_{3}-\sin \beta t_{2} \sin \beta t_{3}+\mathrm{j} z_{c 1} \sin \beta t_{2} \cos \beta t_{3}+\mathrm{j} z_{c 1} \cos \beta t_{2} \sin \beta t_{3}}
\end{aligned}
$$

wherein $z_{c 1}=Z_{c 1} / Z_{0}$ is the normalized closed end impedance, which is the ratio between the closed end impedance $Z_{c 1}$ and the characteristic measurement wave guide impedance $Z_{0} . Z_{c 1}$ will result from the measurement of the transfer functions $T_{12}$ and $T_{34}$ and will have a finite value caused by the measurement imperfections.

The effect of the finite sensing surface of the microphones has been investigated analytically by integrating the pressure distribution of the standing waves inside the duct over the microphone surface. The result is an averaging of the transfer function. This effect is stronger at high frequencies, where the microphone diameter corresponds to a considerable part of the wave length. However, this averaging effect is equal for both transfer functions $T_{12}$ and $T_{34}$. When the transfer function ratio is calculated, the averaging effect vanishes together with the microphone mismatch.

Fig. 6 presents the ratio $T_{c 1}$ between the measured transfer functions $T_{12}$ and $T_{34}$.

In the same way, the ratio $T_{c 2}$ between the transfer function $T_{12}$ and $T_{56}$ is determined. The expression of $T_{c 2}$ is similar to the one of $T_{c 1}$, except that $t_{3}$ is substituted by $t_{5}$ and $t_{4}$ by $t_{6}$. From this expression, the normalized closed end impedance $z_{c 2}=Z_{c 2} / Z_{0}$ will result instead of $z_{c 1}$.

\subsection{Determination of the wave guide damping}

If the wave guide is ideally closed, the transfer function given in Eq. (12) simplifies to

$$
T_{c 0}=\frac{\cos \beta t_{1} \cos \beta t_{4}}{\cos \beta t_{2} \cos \beta t_{3}}
$$

The ratio between the transfer function $T_{c 0}$ and the measured transfer function $T_{c 1}$ is almost unity, except at the poles and the zeros. Apart from the poles and the zeros, the loss factor $\xi$ is the only unknown and will be determined numerically from $T_{c 1}-T_{c 0}=0$. 


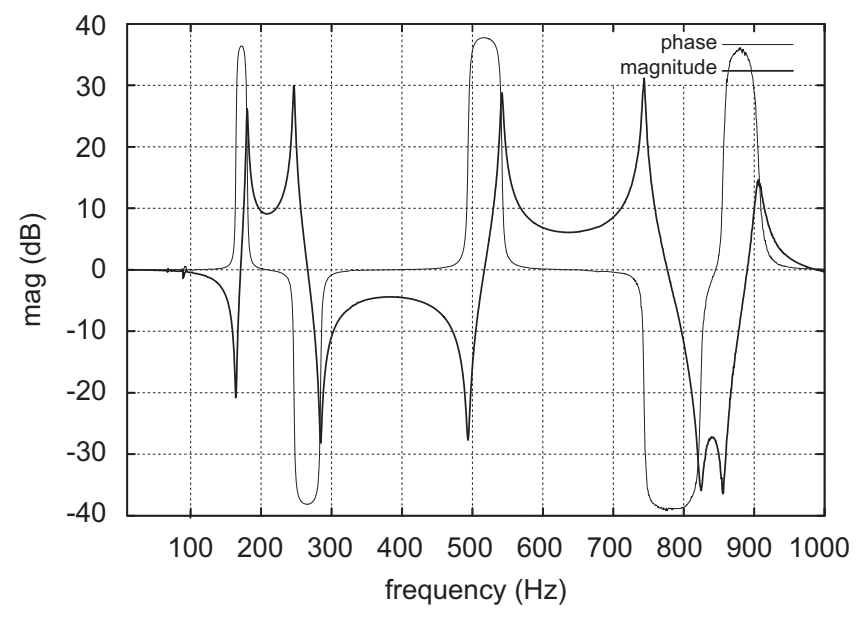

Fig. 6. Ratio between the two transfer functions $T_{c 1}=T_{12} / T_{34}$.

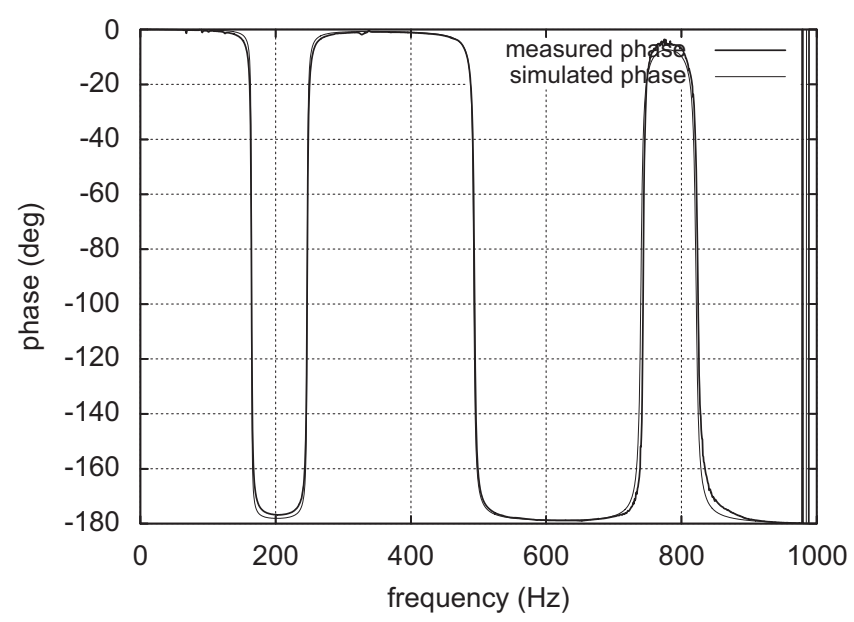

Fig. 7. Comparison between the phase of the measured transfer function $T_{12}$ (thick line) and the phase simulated using expression (7) (thin line).

The obtained loss factor $\xi$ can be introduced as a hysteretic damping for the measured frequency domain. Fig. 7 displays the overlay of the phase of the measured transfer function $T_{12}$ (thick line) and the phase of Eq. (7) (thin line). The damping $\xi$, estimated by the previously described method, is introduced in $\beta=\omega(1-\mathrm{j} \xi)$. Although several damping mechanisms are involved [11], the approximation made by introducing hysteretic damping holds well in a wide frequency band. The deviation between the two phases remains very small.

\subsection{Determination of the closed end impedance}

After introducing the loss factor $\xi$ in the transfer functions $T_{c 1}$ expressed by Eq. (12), the normalized acoustic impedance $z_{c 1}$ of the closed end can be determined from the measured transfer function presented in Fig. 6 . The resulting normalized closed end impedance $z_{c 1}$ is presented in Fig. 8 in thick line. This normalized impedance defines the magnitude measurement range. It must be as high as possible. Expression (12) is quadratic in $z_{c 1}$, resulting in two solutions. One solution corresponds to the direct reference section, the other to the shifted reference section. The solution corresponding to the highest impedance is selected at each frequency point. In the same way, the normalized closed end impedance $z_{c 2}$ is determined from $T_{c 2}$ and displayed in Fig. 8 in thin line. At each frequency, the maximum value of the two resulting closed end impedances will be selected, resulting in the selection function displayed at the bottom of Fig. 8 . When the selection function is low, the value in thick line is selected, otherwise the value in thin line is selected. The resulting normalized closed end impedance $z_{c}$ will be the envelope of the impedances $z_{c 1}$ and $z_{c 2}$. This selection function will be used to determine the unknown impedance, to overlap the singularities. The measurement range, given by the closed end impedance $z_{c}$, reaches $40 \mathrm{~dB}\left(Z_{c} \approx 100 \times Z_{0}\right)$ between 100 and $400 \mathrm{~Hz}$, and $30 \mathrm{~dB}\left(Z_{c} \approx 30 \times Z_{0}\right)$ in the rest of the frequency 


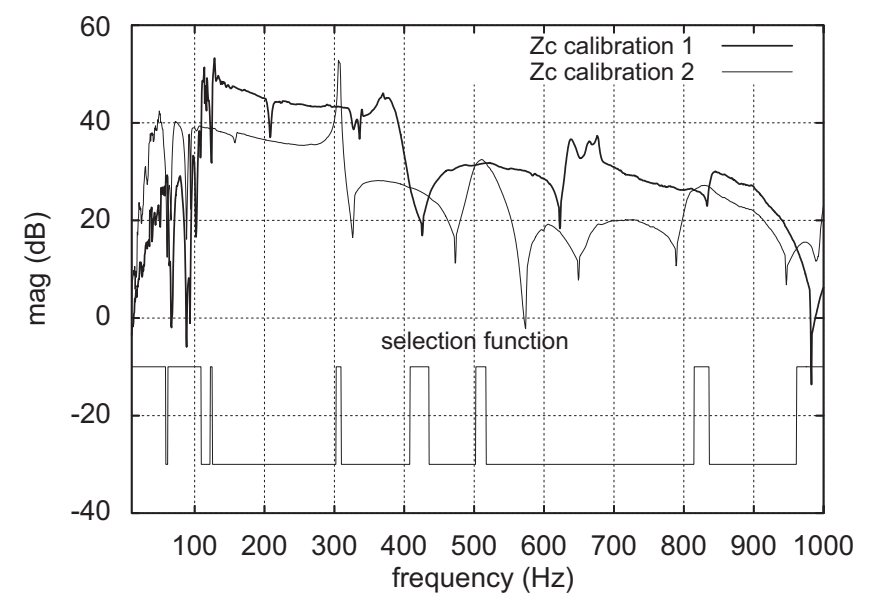

Fig. 8. Resulting closed end impedance amplitude $Z_{c}\left(\mathrm{~dB}\left(\operatorname{ref} Z_{0}\right)\right)$ applying expression (12) to the measured transfer function $T_{c}$.

band. The measurement range can be further extended by adding additional transfer functions at other reference section positions.

\section{Determination of the unknown impedance}

After calibration, the unknown impedance will be determined. For that purpose, the unknown impedance is connected to the wave guide at the reference section and the transfer function $T_{l}$ between the microphones is measured. The unknown impedance $Z_{l}$ will be calculated with respect to each closed end impedance $z_{c 1}$ and $z_{c 2}$ obtained in the previous section.

To remove the sensor mismatch $\delta$, the transfer function ratio $T_{l 1}$ is derived in a similar way as Eq. (12):

$$
T_{l 1}=\frac{T_{l}}{T_{34}}=\frac{\delta\left(Z_{l 1} \cos \beta t_{1}+\mathrm{j} Z_{0} \sin \beta t_{1}\right)}{\left(Z_{l 1} \cos \beta t_{2}+\mathrm{j} Z_{0} \sin \beta t_{2}\right)} \cdot \frac{\left(Z_{c 1} \cos \beta t_{4}+\mathrm{j} Z_{0} \sin \beta t_{4}\right)}{\delta\left(Z_{c 1} \cos \beta t_{3}+\mathrm{j} Z_{0} \sin \beta t_{3}\right)}
$$

wherein $Z_{l 1}$ is the unknown impedance corresponding to $x_{3}$ and $x_{4}$ as distances between the microphones and the reference section. The normalized unknown impedance $z_{l 1}=Z_{l} / Z_{0}$ will result in

$$
z_{l 1}=\frac{\left(\sin \beta t_{1} \sin \beta t_{4}-T_{l 1} \sin \beta t_{2} \sin \beta t_{3}\right)+\mathrm{j} z_{c 1}\left(T_{l 1} \sin \beta t_{2} \cos \beta t_{3}-\sin \beta t_{1} \cos \beta t_{4}\right)}{z_{c 1}\left(T_{l 1} \cos \beta t_{2} \cos \beta t_{3}-\cos \beta t_{1} \cos \beta t_{4}\right)+\mathrm{j}\left(\cos \beta t_{1} \sin \beta t_{4}-T_{l 1} \cos \beta t_{2} \sin \beta t_{3}\right)}
$$

wherein $T_{l 1}=T_{l} / T_{34}$.

Similarly, the normalized unknown impedance $z_{l 2}=Z_{l 2} / Z_{0}$ will result in

$$
z_{l 2}=\frac{\left(\sin \beta t_{1} \sin \beta t_{6}-T_{l 2} \sin \beta t_{2} \sin \beta t_{5}\right)+\mathrm{j} z_{c 2}\left(T_{l 2} \sin \beta t_{2} \cos \beta t_{5}-\sin \beta t_{1} \cos \beta t_{6}\right)}{z_{c 2}\left(T_{l 2} \cos \beta t_{2} \cos \beta t_{5}-\cos \beta t_{1} \cos \beta t_{6}\right)+\mathrm{j}\left(\cos \beta t_{1} \sin \beta t_{6}-T_{l 2} \cos \beta t_{2} \sin \beta t_{5}\right)}
$$

wherein $T_{12}=T_{l} / T_{56}$ and $z_{12}$ is the normalized unknown impedance corresponding to $x_{5}$ and $x_{6}$ as distances between the microphones and the reference section. The resulting normalized unknown impedance $z_{l}$ will be assembled by selecting the proper $z_{l 1}$ and $z_{l 2}$ using the same selection criterion to form the envelope of the normalized closed end impedance $z_{c}$. In this way, the singularities will overlap and the measurement range will be extended.

When the impedance $Z_{l}$ is needed accurately, the ambient pressure and temperature have to be measured. Using expression (2), the characteristic impedance of the measurement wave guide is determined. The unknown impedance results from $Z_{l}=z_{l} Z_{0}$. In this way, the speed of sound has been eliminated from the calibration process.

\section{Test cases}

To demonstrate the capabilities of the presented acoustic impedance calibration method, the open duct end and a closed tube are presented as test cases.

As first test case, the open duct impedance has been measured. The open duct impedance is the other extreme of the closed duct impedance and analytical expressions are available to validate the result [14].

In Fig. 9, the thick line presents the resulting measured open end impedance in magnitude and phase, normalized to the characteristic wave guide impedance $Z_{0}$. The duct end is flanged at the reference section with a flange of $120 \mathrm{~mm}$ diameter. The solution situates between the spherical radiator and the infinite baffle, for which analytical solutions are available. The lower thin line in the amplitude combined with the upper thin line in the phase represents the impedance calculated from the analytical expression of the spherical wave radiator. The other combination of thin lines represent the impedance 


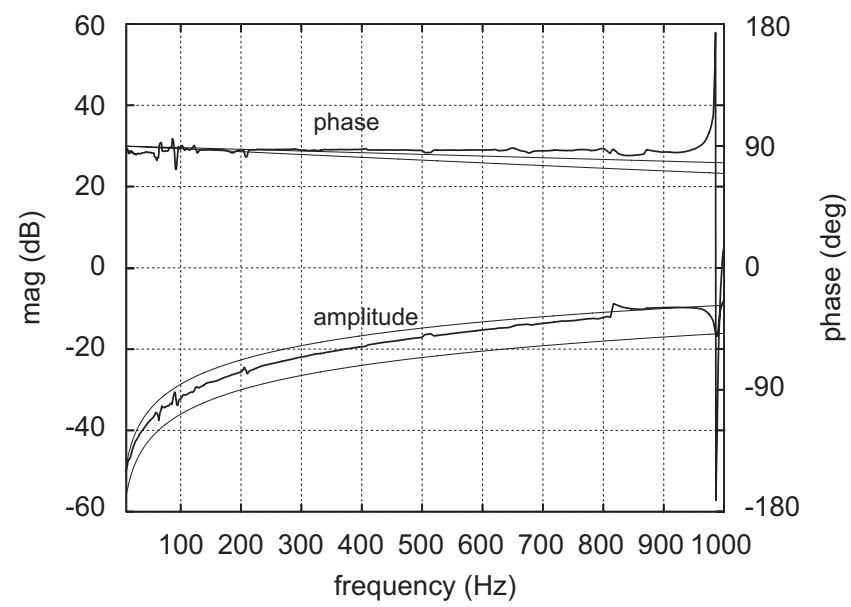

Fig. 9. Resulting open end impedance (thick line) after improved calibration compared to the analytical expressions for the spherical radiator impedance and the piston in infinite baffle (thin lines) $\left(\mathrm{dB}\left(\right.\right.$ ref $\left.\left.Z_{0}\right)\right)$.

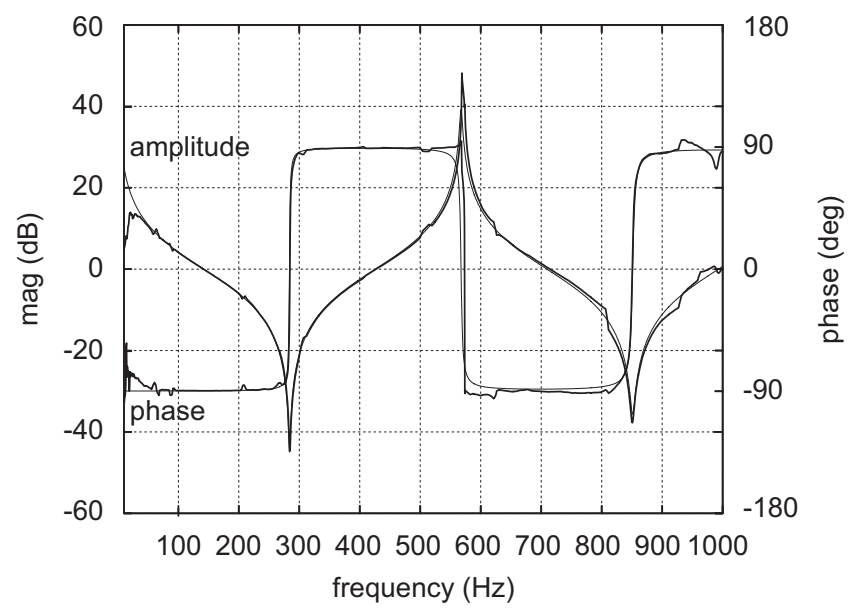

Fig. 10. Measured acoustic impedance (thick line) of a piece of duct of $300 \mathrm{~mm}$ length compared to the analytical solution (thin line) (dB(ref $\left.Z_{0}\right)$ ).

calculated from the analytical expression of a piston in an infinite baffle. The amplitude of the measured open end impedance situates between these two extreme cases. The measured phase situates slightly above the phase of the two cases.

The second test case is a tube with $40 \mathrm{~mm}$ diameter and $300 \mathrm{~mm}$ length, terminated by a hard wall. Fig. 10 presents the measured acoustic impedance in thick line in amplitude and phase, normalized to the characteristic wave guide impedance $Z_{0}$. The analytical expression of the normalized impedance $z$ of a closed tube is given by

$$
z=-\mathrm{j} \cot \gamma l
$$

wherein $\gamma$ is the propagation constant and $l$ the length of the tube. This normalized impedance is also plotted in Fig. 10 in amplitude and phase in thin line. Both plots are consistent. Below $30 \mathrm{~Hz}$, the acoustic impedance measurement is limited by the low response of the pressure sensors at low frequency. This effect is also observed in Fig. 8. The switching of the selection of $z_{l}$ can be observed around 500,800 and $900 \mathrm{~Hz}$. The distance of $80 \mathrm{~dB}$ between the top and the bottom values of the measured impedance gives an indication of the magnitude of the measurement range using the presented calibration method.

\section{Conclusion}

The presented calibration method improves the measurement accuracy of acoustical impedance. It is based on measurements of the hard wall impedance at different positions of the reference section. The deviations caused by measuring the distances between the acoustic centres of the microphones and the reference section, the ambient pressure 
and the temperature are eliminated by the substitution of the wave travelling distances between the respective microphone positions and the reference section by the corresponding wave travelling times. The sensor mismatch vanishes when the ratios between the transfer functions between the microphones at several reference positions are determined. The final hard wall impedance is composed by selecting the maximum of the measured hard wall impedances resulting from each transfer function ratio at each reference section position at each frequency. The same selection mechanism is used to compose the impedance of the unknown impedance. The outcome is an impedance measurement method with a high impedance range in magnitude, ranging from 30 to $40 \mathrm{~dB}$ around the characteristic impedance of the measurement wave guide.

\section{Acknowledgement}

The author thanks the FWO (Fonds Wetenschappelijk Onderzoek) for funding the research project.

\section{References}

[1] J.-P. Dalmont, Acoustic impedance measurement, part I, a review, Journal of Sound and Vibration 243 (3) (2001) $441-459$.

[2] R. Lanoye, G. Vermeir, W. Lauriks, R. Kruse, V. Mellert, Measuring the free field acoustic impedance and absorption coefficient of sound absorbing materials with a combined particle velocity-pressure sensor, Journal of Acoustical Society of America 119 (5) (2006) $2826-2831$.

[3] P. Dickens, J. Smith, J. Wolfe, Improved precision in measurements of acoustic impedance spectra using resonance-free calibration loads and controlled error distribution, Journal of Acoustical Society of America 121 (3) (2007) 1471-1481.

[4] M.G. Prasad, A four load method for evaluation of acoustic source on a duct, Journal of Sound and Vibration 114 (2) (1987) 347-356.

[5] L. Desmons, J. Hardy, Y. Auregan, Determination of the acoustic source characteristics of an internal combustion engine by using several calibrated loads, Journal of Sound and Vibration 179 (5) (1995) 869-878.

[6] H. Bodén, On multi-load methods for determination of the source data of acoustic one-port sources, Journal of Sound and Vibration 180 (5) (1995) $725-743$.

[7] ISO 10534, Determination of sound absorption coefficient and impedance in impedance tubes, International Organisation for Standardization, Case postale 56, Genève, 20, 1998.

[8] H. Bodén, M. Åbom, Influence of errors on the two microphone method for measuring acoustic properties in ducts, Journal of Acoustical Society of America 79 (2) (1986) 541-549.

[9] B.F.G. Katz, Method to resolve microphone and sample location errors in the two-microphone duct measurement method, Journal of Acoustical Society of America 108 (5) (2000) 2231-2237.

[10] V. Gibiat, F. Laloë, Acoustical impedance measurements by the two-microphone-three-calibration (TMTC) method, Journal of Acoustical Society of America 88 (6) (1990) 2533-2545.

[11] A.D. Pierce, Acoustics: An Introduction to its Physical Principles and Applications, McGraw-Hill, New York, 1981 (Chapter 10).

[12] E.J. Scudrzyk, Foundations of Acoustics, Springer, Berlin, 1972, p. 283.

[13] H.P. Neff Jr., Basic Electromagnetic Fields, Harper \& Row, New York, 1987, p. 463.

[14] L.L. Beranek, Acoustics, McGraw-Hill, New York, 1954, pp. 36, 119. 